\title{
MODELING DISEASES: PREVENTION, CURE AND MANAGEMENT
}

\author{
Christine S.M. Currie \\ Mathematical Sciences \\ University of Southampton \\ Highfield \\ Southampton, SO17 1BJ, UK
}

\author{
Thomas Monks \\ NIHR CLAHRC Wessex \\ University of Southampton \\ Highfield \\ Southampton, SO17 1BJ, UK
}

\begin{abstract}
Diseases, like death and taxes, seem an inevitable part of life, with much of modern medicine acting to prevent, cure or manage them effectively. Modeling of diseases began over a century ago and has been one of the success stories of applied mathematics and, more recently, computer simulation. In this article, we describe modeling of both communicable and non-communicable diseases, and provide a review of the relevant literature. Two case studies are discussed, the first describing a modeling study of tuberculosis and HIV and the second capacity planning for stroke services. Our aim is to instigate a discussion of how modeling should be used in the future to answer the most up-to-date questions about global health, particularly those of disease prevention.
\end{abstract}

\section{INTRODUCTION}

We focus here on the modeling of diseases from a public health or epidemiology viewpoint rather than that of mathematical biology. Our aim is not to model individual biological processes but instead to consider how to describe the impact of diseases on populations. The nature of diseases is such that there is a natural split into communicable and non-communicable diseases. While some of the modeling methods are applicable to both disease types, on the whole, the focus of the modeling questions is very different.

In what follows we describe a number of different modeling approaches, discrete event simulation (DES), system dynamics (SD), agent based modeling (ABM), differential equation models (also referred to as compartmental models) and Markov chains. DES, ABM and Markov chain models are stochastic, meaning that they make use of pseudo-random numbers to mimic the uncertainty inherent in real processes. SD and differential equation models are typically deterministic, producing exactly the same output for the same set of input parameters. The different modeling paradigms can also be distinguished by whether they are at the level of the individual, as is the case for DES and SD models or if they are population-based, such as SD, differential equation models and Markov chains.

When modeling communicable diseases, a key factor is the mode of transmission, and this can play a strong part in dictating the structure of the model. Many diseases are transmitted via close contact between an infectious individual and a susceptible individual but others require either an insect vector, such as a mosquito in the case of malaria or dengue fever, or are perhaps transmitted via contaminated water. Reducing the effectiveness of the mode of transmission by prophylaxis, vector control or even by providing clean water, as in the famous case of John Snow reducing cholera deaths in London (Paneth et al. 1998), can be one of the key mechanisms for halting an epidemic. Modeling can be an important way of determining which of the control measures is most effective; particularly as it enables account to be taken of the reduction of future cases of the disease due to reduced transmission.

The mathematical modeling of communicable diseases has a long history, beginning in the early 20th century with work by Kermack and McKendrick (1933), Bartlett (1957) and Bailey (1950), who principally 


\section{Currie and Monks}

consider stochastic models. The focus shifted from stochastic to deterministic models in the 1990s as Anderson and May (1993) used differential equation models to describe the natural history of diseases. These models are incredibly successful at a population-level for a large number of communicable diseases and much of the public health and computational biology literature still features them extensively. The mathematics is such that, even with some of the more complex differential equation models, it can be possible to derive analytical results about the equilibrium states and this can be attractive as it removes the messiness of stochastic or simulation models. Differential equation models are very much akin mathematically to SD models but tend to approach the modeling from a different perspective.

Simulation studies of non-communicable diseases often focus on the delivery of services; for example, optimization and evaluation of the cost effectiveness of the operations and logistics of patient care. Models typically take a DES, SD or Markov Chain approach. Computer simulation has been used extensively within healthcare and interested readers can find models from mental health (Lebcir et al. 2017; Thompson et al. 2014; Cepoiu-Martin and Bischak 2017), cancer screening (Comas et al. 2016; Jansen and Zoetelief 1997; Brailsford et al. 2012), stroke (Monks et al. 2017; Lahr et al. 2017), diabetes (Chalk et al. 2012; Davies et al. 2000) and heart disease models (Vasilakis et al. 2007; Mustafee et al. 2012).

The paper is split into two main parts, the first discusses the modeling of communicable diseases, providing a review of different modeling studies in 2 and then using a case study of tuberculosis (TB) to demonstrate the complexities of disease modeling and the factors that need to be taken into account when setting up communicable disease models. The second section covers non-communicable diseases and gives advice on setting up modeling studies before focusing on a case study of operational modeling for patients suffering from a stroke.

\section{MODELING COMMUNICABLE DISEASES}

Communicable diseases can be transmitted in different ways and at different rates and the transmission mechanism is a key part of the models. As a result, we organize this section into five parts:

- Modeling outbreaks: these models focus on transient epidemics, with rapid increases in the number of infected people due to a high probability of transmission and fast disease progression. The research is almost exclusively on influenza but also includes models of bioterrorism.

- Modeling endemic diseases: an infectious disease is said to be endemic if there is a relatively constant base level of incidence of the disease within the population.

- Modeling sexually transmitted diseases: these diseases call for a particular type of model as they rely on a sexual contact network for transmission.

- Modeling vector-borne diseases: transmission takes place via vectors rather than directly.

- Modeling water-borne diseases: transmission takes place as a result of coming into contact with contaminated water either directly through drinking or washing, or indirectly by eating food that has been exposed to it.

The split has been chosen to reflect differences in the types of models that are used to describe transmission rather than their biological nature, as is done in, e.g., Keeling and Rohani (2007).

The historical literature has relied heavily on deterministic differential equation models and packages such as Berkeley-Madonna (https://berkeley-madonna.myshopify.com/) have made it straightforward to quickly build relatively complex differential equation models of infectious diseases. Such models are useful in population based studies but fall down when the numbers involved are too small for average flows to be meaningful. They also have difficulty modeling situations where an individual's characteristics are important in determining progression or transmission as in these cases, many different states are needed to account for the differing risks and the lack of homogeneity in the general population. For these reasons, $\mathrm{ABM}$ is becoming more prevalent and we concentrate our discussion on these models. DES models are used for communicable diseases but these are often more focused on the management of the resources 


\section{Currie and Monks}

used to control the disease. For example, Worth et al. (2010) investigate the effect of scarce resources on contact tracing for pertussis and Harper and Shahani (2003) use DES to estimate the future costs of caring for HIV/AIDS patients.

\subsection{Modeling Outbreaks}

Perhaps spurred on by news reports of a possible influenza pandemic, the Winter Simulation Conference saw a large number of papers on pandemic influenza between 2009 and 2011. This may also have been helped by Epstein's opinion article in Nature, which advocates for the use of ABMs in modeling pandemics (Epstein 2009). The World Health Organization (WHO) defines a pandemic to be "the worldwide spread of a new disease", and it is these severe outbreaks that have been the focus of much of the modeling research.

The standard control interventions for pandemic influenza are vaccination and isolation, where isolation can take many different forms, e.g., school closures or home confinement. The level of detail required in describing the population is dependent on the nature of the interventions being tested. For example, when examining the optimal distribution of the influenza vaccine among different age groups, Özaltin et al. (2014) by necessity include age-specific mixing in their model. Different categories of mixing groups are included in the majority of the models, allowing for mixing being non-homogeneous in the population. This gives a more realistic view of how transmission takes place. Paleshi et al. (2011) include households, daycare centers, schools, work places and community. They compare two isolation strategies: school closures and home confinement. Setting up an individual-based model of infection requires estimates both of who individuals interact with and how close that contact is: casual contact, close contact, indirect contact (through bacteria left behind on a surface that both individuals come into contact with). We discuss contact networks in more details below when describing sexually transmitted diseases.

Hu et al. (2014) describe an alternative method of transmission that they hypothesize is a good description of bioterrorism. Their work aims to distinguish between an epidemic outbreak and a bioterrorism attack and assumes that transmission in a bioterrorism attack only takes place if an individual is in close proximity to the source of the biological agent. This differs from transmission in a "standard" epidemic, by including no transmission away from the attack site.

The natural history of the disease is often included at a fairly coarse level of detail. For example, an SEIR (Susceptible-Exposed-Infected-Recovered) model is used by Jung et al. (2017) to describe progression through MERS (Middle East Respiratory Syndrome). Other authors may split states to add detail, where this helps the modeling; for example, dividing the infected stated into symptomatic and asymptomatic states (Paleshi et al. 2011).

The ABMs developed are often very large, with millions of individuals. For example, Ma et al. (2011) describe a model of New York City for the 2009 H1N1 pandemic with 18 million nodes and 1 billion edges and Aleman et al. (2009) use an ABM with nearly 5 million individuals to describe the Greater Toronto Area, Canada. Experimenting with such models requires high performance computing to reduce run times to reasonable levels and Aleman et al. (2009) describe a parallelization scheme that is needed to reduce the experimentation time to a reasonable level. An alternative to these large models but with some of their advantages over differential equation models is the scalable stochastic model of infectious disease propagation described in Sanchez and Sanchez (2015). This uses Ebola rather than influenza to demonstrate how it works but the principles are similar. Their aim is to "avoid aggregation, include stochastic behaviors, avoid unnecessarily restrictive modeling assumptions, and eliminate the artifacts associated with time-stepped modeling". The model they build is computationally efficient, as computation only occurs when an event takes place rather than in every time step of the simulation.

\subsection{Modeling Endemic Diseases}

Endemic diseases are often regarded as being under control and modeling exercises tend only to be instigated when the incidence rates start to increase or as a result of political pressure such as the introduction of the 


\section{Currie and Monks}

Millennium Development Goals (MDG). One of the more important endemic diseases is tuberculosis (TB), which saw an increase in modeling studies in the late 1990s and early 2000s. This may be a result of TB being the target of one of the MDGs but TB also saw a rise in incidence in the 1990s and early 2000s due to two exacerbating factors: multi-drug resistance and the effect of the HIV epidemic. Both have led to increases in TB incidence in some areas of the world. Sub-Saharan Africa in particular has been suffering from a co-epidemic of TB and HIV, where the interaction between the two diseases comes about because an untreated HIV infection increases an individual's susceptibility to developing TB disease. We consider TB in detail in Section 3 and so do not discuss it further here.

Particularly topical at the moment is the challenge faced in many richer countries of dealing with the opioid crisis. While not strictly a communicable disease, much of the modeling draws heavily on ideas from infectious disease modeling. Benneyan et al. (2017) consider three different styles of models that consider population-level dynamics (typically differential equation models); geographic-level topology spread (typically models based on cellular automata models); and individual-level influence across networks (using ideas of diffusion across networks).

\subsection{Modeling Sexually Transmitted Diseases}

Recent models of sexually transmitted diseases (STD) tend to be heavily dependent on the choice of social network used to describe contact between individuals. Possibly the earliest version of such a network model was STDSIM, developed in the late 1990s and used in many subsequent modeling studies of HIV (der Ploeg et al. 1998). In more recent years, the network models have become more complex to incorporate real data from the populations under study. For example, Rutherford et al. (2016) describes a body of work around modeling the HIV epidemic in Vancouver, which includes a network model of injecting drug users and female sex workers, with the aim of assessing the effectiveness of different control strategies.

In disease modeling studies, the network model will generally consist of a set of vertices (individuals) connected by edges (contacts), where it is assumed that the contacts are such that if two vertices are connected by an edge, a transmission event could take place. An obvious important feature of a network is how well individuals are connected, measured by the degree distribution - the probability distribution for the number of edges each node in the network has. The edges in the network may have different weights, corresponding to different types of partnership; for example, in modeling a network of heterosexual contact, Enns and Brandeau (2011) suggest three levels: 0, no contact; 1, spousal partnership; 2, non-spousal partnership. This allows different probabilities of transmission for different types of contact. In the absence of extensive datasets such as those collected in Vancouver, setting up a contact network in a model can be difficult. Building complex models incorporating social networks requires a large team of people drawing on expertise in ethnography, statistics and computer science as well as mathematics and medicine. Enns and Brandeau (2011) suggest an alternative for generating a contact network that draws on only a few summary statistics about the model population. A similar generator of a contact network is reported in Zhang et al. (2012).

Incorporating contact networks allows testing of interventions that break transmission connections between individuals, such as condom use or needle exchange programs. It can also enable evaluation of the impact of so-called superspreaders (Lee et al. 2013). While not aimed at STD modeling, this work examines how the topology of a network might affect the evolution of an epidemic.

Not all simulation models of STDs are ABMs. Kok et al. (2015) use SD to determine optimal resource allocations among targeted and routine testing programs, with the aim of reducing the incidence of new

HIV infections. SD is an obvious choice for modeling the feedback between increasing the number of HIV diagnoses and reducing the number of new HIV infections, assuming patients adhere correctly to treatment. Incorporating the SD model in the optimization of resource allocations is a straightforward way of ensuring that the full benefits of early detection are accounted for. 


\subsection{Modeling Vector-Borne Diseases}

Vector-borne diseases account for more than $17 \%$ of all infectious diseases. Transmission is made possible by vectors, typically blood-sucking insects, which ingest the micro-organisms responsible for the disease during feeding from an infected host. These are injected into a susceptible host in a subsequent blood meal. The presence of the vector makes vector-borne diseases more complex to model as it is necessary to consider the dynamics of both the vector and host populations.

As with other communicable diseases, early modeling used differential equation models to describe the natural history of the disease, and Ross's models of malaria are among the earliest examples of mathematical modeling of diseases (Ross 1911). Ross assumes a simple structure for both the hosts and vectors, each consisting of just two states: susceptible and infected. A full review of differential equation models for malaria is given in Mandal et al. (2011), who claim this to be the main modeling methodology in the area.

Recent work at the Winter Simulation Conference on vector-borne diseases has been more focused on dengue fever which, although less deadly than malaria, has become an increasing public health problem. Dengue is a mosquito-borne disease and occurs in more than 100 countries in tropical and subtropical regions. Recent medical advances in vaccine development has prompted some of the recent modeling work to focus on vaccination strategies (e.g., Knerer et al. (2015), Perkins et al. (2018)). ABM is a natural tool for modeling these vector-borne diseases and can enable geographical factors to be incorporated into the model (de Lima et al. 2016; Isidoro et al. 2011). This can be especially beneficial when determining the effectiveness of vector control policies.

\subsection{Modeling Water-Borne Diseases}

Given the importance of water-borne diseases, which include cholera and dysentery, there has been relatively little simulation modeling in the area with the core modeling research using differential equation models (Codeço et al. 2008). Crooks and Hailegiorgis (2013) demonstrate the clear benefits of using an ABM structure to describe the spread of cholera in a refugee camp. Their model, which includes a relatively detailed picture of the spatial environment in the Dadaab refugee camps, includes a hydrological model to determine water movements as well as a relatively simple model of people's movements around the camps. Their tests explain how rainfall can lead to peaks in the incidence of cholera and show how an infection in just one borehole at the camp can spread. Similar work has been carried out for a gastro-intestinal disease outbreak by Okhmatovskaia et al. (2010). These models are complex and draw on a wide range of data and expertise.

\section{CASE STUDY: MODELING TUBERCULOSIS}

TB is transmitted by the exchange of Mycobacterium tuberculosis (mTB) bacilli through the air, usually via a patient coughing, sneezing, talking, spitting or singing, and close social contact is required for successful transmission. As a result, the transmission probability can be very low as Kenyon et al. (1996) show in a study of passengers on a long-distance airplane flight. The natural history of TB differs greatly from that of the acute infections. First, only about $10 \%$ of those infected with the mTB bacilli eventually develop disease. Second, the time interval between infection and disease onset can be long: disease can occur within 6 months of an initial infection or many years thereafter through either reactivation of a previous infection or following reinfection. In the majority of cases, death from other causes occurs before reinfection or reactivation; the exception being for HIV-positives where rates of reactivation are much higher.

A diagram showing a possible model of progression through TB disease is shown in Figure 1. Few authors include all of the states shown in this figure, with the level of detail determined by the questions that they are seeking to answer. The majority of models will however include at least four states: (1) uninfected or susceptible to disease; (2) possessing a latent mTB infection; (3) suffering from active TB disease; (4) undergoing treatment for TB disease. 


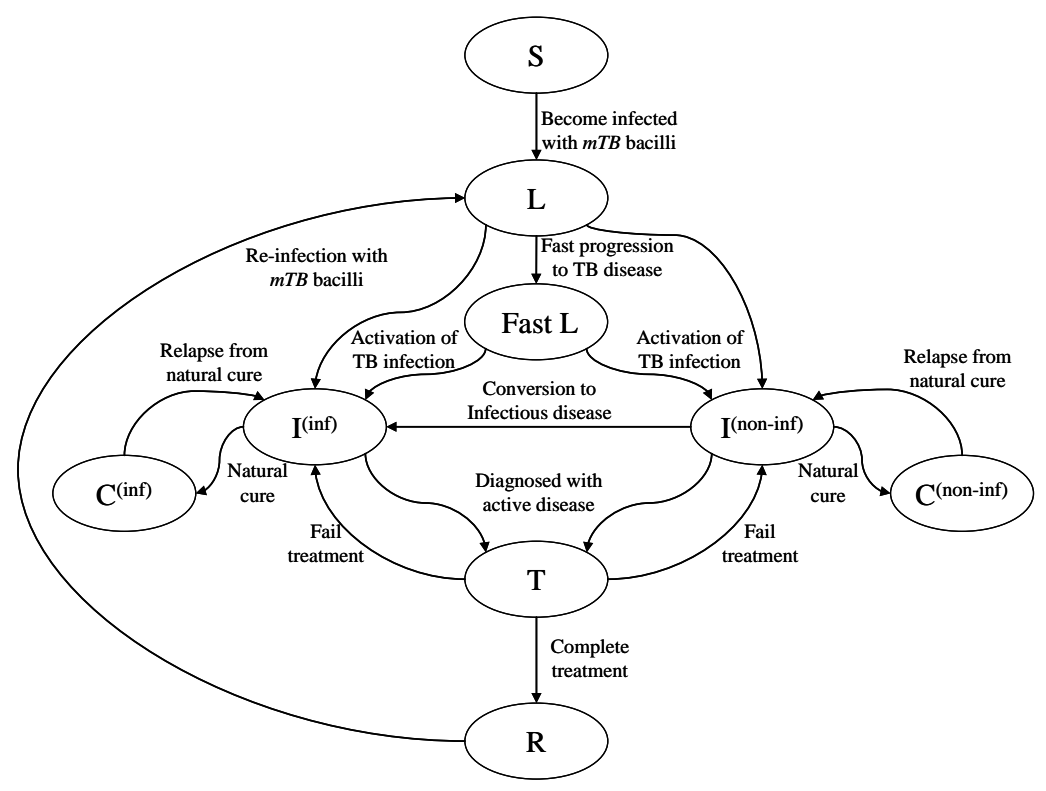

Figure 1: Progression through TB disease, where S denotes uninfected or susceptible persons; L those with a latent mTB infection; Fast L those who will progress quickly to active disease; I(inf) persons with active, infectious (smear sputum positive) disease; I(non-inf) persons with active, non-infectious (smear sputum negative) disease; $\mathrm{C}$ (inf) and $\mathrm{C}$ (non-inf) persons who have spontaneously cured from active infectious or non-infectious disease; $\mathrm{T}$ persons undergoing treatment for active TB disease and $\mathrm{R}$ persons who have recovered from treatment for active TB disease. Individuals may die from any state (not shown).

Research in the modeling literature has considered the relative importance of the three disease mechanisms - primary infection, reactivation and reinfection - as assessing their relative impacts is important for predicting the effect of control policies. Kasaie et al. (2014) build an ABM simulation of TB transmission in which different mTB strains are exchanged among individuals in the population. This enables study of clustering dynamics in relation to disease incidence, diversity of circulating strains, sampling coverage, and study duration. Similar ideas and methods are considered by Murray (2002).

There has also been some considerable focus in the literature on strategies that involve identifying close contacts of TB patients as this tends to be an effective strategy for identifying follow-on infections and it is these modeling studies that tend to use stochastic simulation models rather than differential equations. Individual-based simulation models, e.g. DES and ABM, allow heterogeneity in transmission to be modeled much more easily than the deterministic compartmental models that have often been used in TB modeling.

The paper by Mellor et al. (2010) takes the first step to doing this by including a household structure, with an increased likelihood of transmission between persons in the same household. The model, which is based on data from Zimbabwe, and therefore has a study population with a relatively high prevalence of HIV, is able to reproduce clustering of TB and HIV cases in households, even without explicitly modeling the transmission of HIV. The role of contact networks is further explored by Kasaie et al. (2013) who use an ABM simulation to determine the impacts of three levels of network: close contacts (household members), casual contacts (neighbors), and random contacts (community).

Murray (2002) aims to explain results of DNA fingerprinting of mTB strains and uses a similar network structure to Kasaie et al. (2013). Individuals in the model are also given a vector of attributes including age, HIV status and the presence or not of a latent mTB infection. These attributes change their risks of infection and, once infected, their risk of transmitting an infection, as well as affecting their progression through clinical disease. The results show that these risk factors help to determine the distribution of mTB 


\section{Currie and Monks}

strains in the population and that these population characteristics seem more important in determining the cluster size than the success of control measures or any strain-specific characteristics.

The spread of HIV infection in the 1980s and 1990s led to a dramatic increase in TB incidence in eastern and southern Africa, as a result of the increased risk of TB experienced by HIV-positive individuals. Because of these increases, policies for controlling the TB incidence were strengthened and adapted to take account of HIV as a risk factor for TB; for example, by integrating TB control activities with those of HIV programs and including provision for HIV/AIDS care in TB clinics (de Cock and Chaisson 1999).

Long et al. (2008) provide a mathematical treatment of these co-epidemics, using data from India in their models. Their results show that coordinated treatment activities are necessary and that exclusively treating just one of the two diseases can be a particularly poor strategy. The challenge in building dynamic models of TB and HIV is in deciding on the most appropriate level of detail for the natural history of HIV and TB in the model: too much detail complicates the model, making the subsequent results less transparent and often harder to accept. A further obvious drawback of including increasing amounts of detail into a model is that unless data for all of the assumptions are available, extensive (and time-consuming) sensitivity analyses of the model parameters are required in order to justify the models conclusions. However, the more detailed a model the more realistic it can seem, which can make it more acceptable to users.

Mellor et al. (2010) use a relatively simple, statistical model of HIV that drives a more complex transmission model of TB, choosing this version as the aim of the study was to develop new guidelines for contact tracing for TB in areas with a severe HIV epidemic. The focus of the research questions will often dictate where and how the complexity is included.

\section{MODELING NON-COMMUNICABLE DISEASES}

\subsection{General Advice}

Before we provide specific advice for methods for computer simulation of non-communicable disease pathways, we provide some straightforward advice on project setup.

\subsubsection{Clarify modeling objectives and what the clinical team wants to achieve}

Unlike the manufacturing or defense sectors, healthcare managers and clinicians are less likely to be familiar with computer simulation. Our advice is to carefully guide healthcare professionals through the basics of computer simulation while establishing modeling objectives. A tactic that appears to work well is to present a computer simulation study from an analogous, but indirect application domain (for example, a call center model from the service sector).

Establishing clear objectives can be a challenge, and modelers should beware vague objectives such as 'minimize cost' or even potential misguided objectives such as maximize utilization. It is recommended that modelers investigate how the performance of the system is reported; potentially by reviewing routinely produced reports for the system.

\subsubsection{Identify the patient populations to model}

In most disease pathways, the patient population will be sub-dividable. Initial conceptual modeling should focus on identifying which populations need to be modeled and if detail is needed to subdivide into different classes. As an example, in a model of a dementia pathway a modeler must establish if it is it all types of dementia diagnosis that need inclusion or is it only an Alzheimer's diagnosis? Are there any other type of patient that use the same services; for example, patients with mild cognitive impairment as opposed to dementia? Do different ages groups have different processes? For example young onset dementia may be more difficult to diagnoses and require more appointments and increased use of resources. 


\section{Currie and Monks}

\subsubsection{Use the initial meeting to sketch out an overview of the disease pathway}

Clinical teams are often very busy and while they will do their best or meet with modelers, it is often more limited than ideal. We therefore advise modelers to sketch an overview of a clinical pathway in the very first project meeting.

\subsubsection{Spend some time observing how the operations of the disease pathway are managed}

The description of the disease pathway obtained in the initial meeting may be idealized. It is advised that modelers spend at least a few hours in the one or more services in the real pathway to observe how it operates and to interview on-the-ground workforce. Observations of the system under pressure are useful to understand the complexities of the pathway and will reveal aspects that have been missed or that operate differently from described. Figure 2 illustrates a cardiac surgery pathway at a large hospital that operated on patients from a network of smaller hospitals in the UK. The diagram itself was primarily constructed in an initial meeting with a group of cardiac surgeons. Observation of the system and shadowing of specialist cardiac nurses revealed two differences from the idealized model. Firstly, patients could deteriorate and bounce back from post-operative care to higher levels of care. Secondly, in times of pressure, the post operative beds were used flexibly to accommodate patients waiting for surgery and those who had already undergone surgery.

\subsubsection{Meet with the data controller for the pathway}

Access to data within healthcare modeling can sometimes be difficult due to data protection regulations and high demand for healthcare data analysis within the organizations. It is recommended that modelers seek an early meeting with the individuals that manage the data within the organization. This provides an opportunity to explain the objectives of the project and computer simulation. It also allows the modelers to find out what data are collected routinely and help shape what is to be modeled.

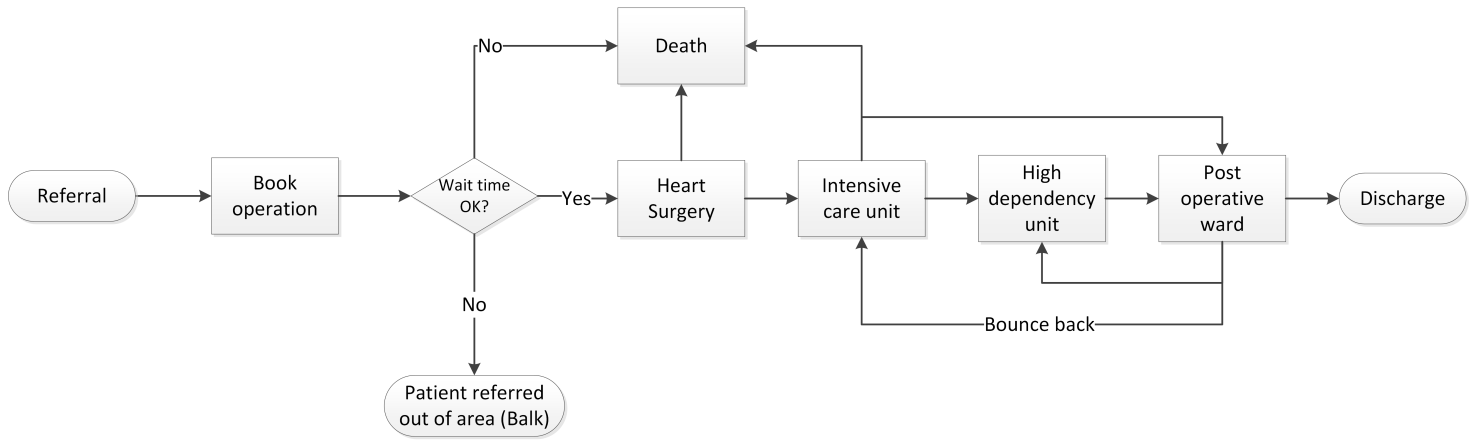

Figure 2: Process Flow Diagram of Cardiac Surgery Pathway.

\subsection{Data Sources}

Health services routinely collect a large volume of data on the delivery of patient care and treatments. These data sources are often disparate within and across care organizations. For example, the diagnosis and delivery of care for patients with Dementia may be split across primary care (i.e. a family doctor), acute hospital care (e.g. brain scanning services, specialist wards and emergency departments within a large hospital), community-based care (i.e. outpatient clinics and home visits), informal care (e.g. services provided by the Alzheimers Society), and institutional care (e.g. a nursing home). The range of services accessed by patients suffering from long-term diseases means that parameterizing simulation models of 


\section{Currie and Monks}

full pathways is often hampered by issues in data linkage, information governance and knowledge of what is available.

\subsection{Input Data}

To model disease pathways a simulation modeler will need access to the following types of input data.

\subsubsection{Demand data}

For example, the date and time that emergency medical services were called regarding a stroke or the time that a cancer patient was referred for treatment. Data on demand may breakdown into different populations. For example, individuals with heart disease will undergo different forms of cardiac surgery: atrial valve replacement, mitral value replacement and coronary artery bypass graft. An alternative approach is to sub-divide the population into scheduled elective and unexpected urgent operations. For stochastic simulation studies, particularly those that involve an urgent and emergency healthcare element it is useful to obtain a substantial time series to estimate accurate inter-arrival time distributions for these populations.

It is also worth noting that if a disease pathway involves outpatient clinics or any form of appointment system then demand data may contain appointments that were missed (typically labeled Did Not Attend or DNA). It is important that modeling take account of these missed appointments, as in some cases, such as mental health, rates can be high.

\subsubsection{Process time data}

These data describe a process duration. From our heart disease example, this would include the duration of cardiac surgery in an operating theater and an associated stay in an intensive care unit or other wards. Outside of a hospital led service process data might describe the length of a memory clinic appointment for a patient with suspected dementia or length of a home visit. Unlike demand data it is not always necessary (or possible) to obtain a large-scale sample of process time data and a smaller sample can be used to estimate model inputs. In these cases, modelers may need to consider input uncertainty (Song et al. 2014).

\subsubsection{Patient routing data}

Disease pathways straddle multiple organizations and services. This means that simulation models of a disease pathways are likely to need estimates of routing and its associated variability. For example, in the cardiac surgery example, not all patients require high-dependency step down care and will move directly to the post-operative ward. Similarly, a proportion of patients will deteriorate and be routed back from post-operative care to a high-dependency ward while the remaining proportion will be discharged.

\subsubsection{Resource data}

The simplest data required for simulation models are quantitative data on the number of resources available. In the cardiac surgery example, this would include the number of beds within the intensive care high dependency and post-operative wards. These resources may be pooled or may be specialized in some way; for example, split up in the case of gender specific accommodation. Depending on the modeling objectives this may need to extend to workforce.

\subsubsection{Disease progression data}

In some disease areas patients may be undergoing treatment while their disease state is progressing. As an example, consider individuals with diabetic retinopathy: a complication of diabetes that leads to blindness. It is essential to identify and treat retinopathy at a certain stage of the disease. Hence, when modeling screening and treatment policies it is necessary to model both the delivery of care and the progression of 


\section{Currie and Monks}

the retinopathy (Chalk et al. 2012; Davies et al. 2000). Models of disease progression and aging may also need to take account of mortality, possibly adjusted for age and stage of disease. Estimates of input data to model disease progression data is best sourced from the clinical literature.

\subsection{Data Issues}

Perhaps the most common data deficiency encountered when building simulation models that straddle multiple services and organizations is the lack of a time-stamp to indicate when a patient is ready to be discharged (here used to describe the time when a patient is both medically fit and has a discharge plan in place). This means that routine data often reflect total length of stay as opposed to treatment time and departure queueing time. The simplest way to mitigate this issue and estimate the distribution and parameters of treatment time is to use expert opinion. For example, by asking what percentage of length of stay is spent waiting for transfer. If this option is taken modelers need to be very specific about which patients they are referencing and should also make extensive use of sensitivity analysis. The second option is to use model calibration techniques. That is, if a sample of the performance measure of interest is available, the input parameters are systematically varied until outputs agree with empirical observations. These approaches lead to multiple sets of input parameters that each provide matching output. Results should then be presented for each of these sets to adequately represent the uncertainty in the inputs.

A secondary problem is that many patients do not queue in the usual sense when a bed in a specialist ward is unavailable, they are instead admitted to an alternative ward. There is often insufficient data to identify how these patients are repatriated, for example, first in first out, if decision rules are place to reject repatriation if only a short amount of treatment time remains, or indeed if patients resident on the specialist ward who are waiting to be transferred to rehabilitation are moved to an alternative ward to make space. Of course, some of this can be handled by assumptions agreed with simulation clients.

\section{CASE STUDY: STROKE SERVICES CAPACITY PLANNING}

\subsection{Background to Stroke Pathways}

Stroke is major cause of disability internationally and accounts for approximately 1 in every 9 deaths worldwide. The World Health Organization estimates that fifteen million people suffer a stroke each year. The cost of stroke is estimated to be $2 \%$ to $4 \%$ of total healthcare costs worldwide.

Figure 3 illustrates a typical stroke treatment and rehabilitation pathway. After the onset of a stroke the best place for a patient is an Acute Stroke Unit (ASU). An ASU is a dedicated ward for patients with suspected stroke comprising of a multi-disciplinary team of specialist physicians and nurses. Acute care often involves an assessment of the patient, identification of the type of stroke suffered and, in some cases, early rehabilitation. Length of stay on an ASU is typically seven days for those with a final diagnosis of stroke. There are some caveats, however, as admission to an ASU might be severely delayed due to lack of beds. This will be for a number of reasons: the natural variation in stroke admissions and treatment duration, the inevitable overspill of patients from other wards in a highly utilized hospital and transfer delays of patients to rehabilitation. Where direct admission is not possible stroke patients become outliers and are admitted to another hospital ward. Unless the hospitals stroke consultants visit their outlier patients, these patients will usually not have further access to a stroke specialist and will be seen by the resident, non-stroke, specialist on their ward.

In the UK acute care is often based in a large acute hospital while rehabilitation capacity is split over a number a of smaller community-based hospitals. Length of stay in rehabilitation is substantially longer than an acute stay (average 30-40 days). Patients receive a number of treatments to aid rehabilitation from their stroke in this time including physical and speech therapy. Discharge from rehabilitation can be substantially delayed as patients may require special care packages from social services (a separate organization from the UK National Health Service) to be in place before they go home; a place in a care home or simply because there is a family dispute about how the patient will be cared for at home. In a 


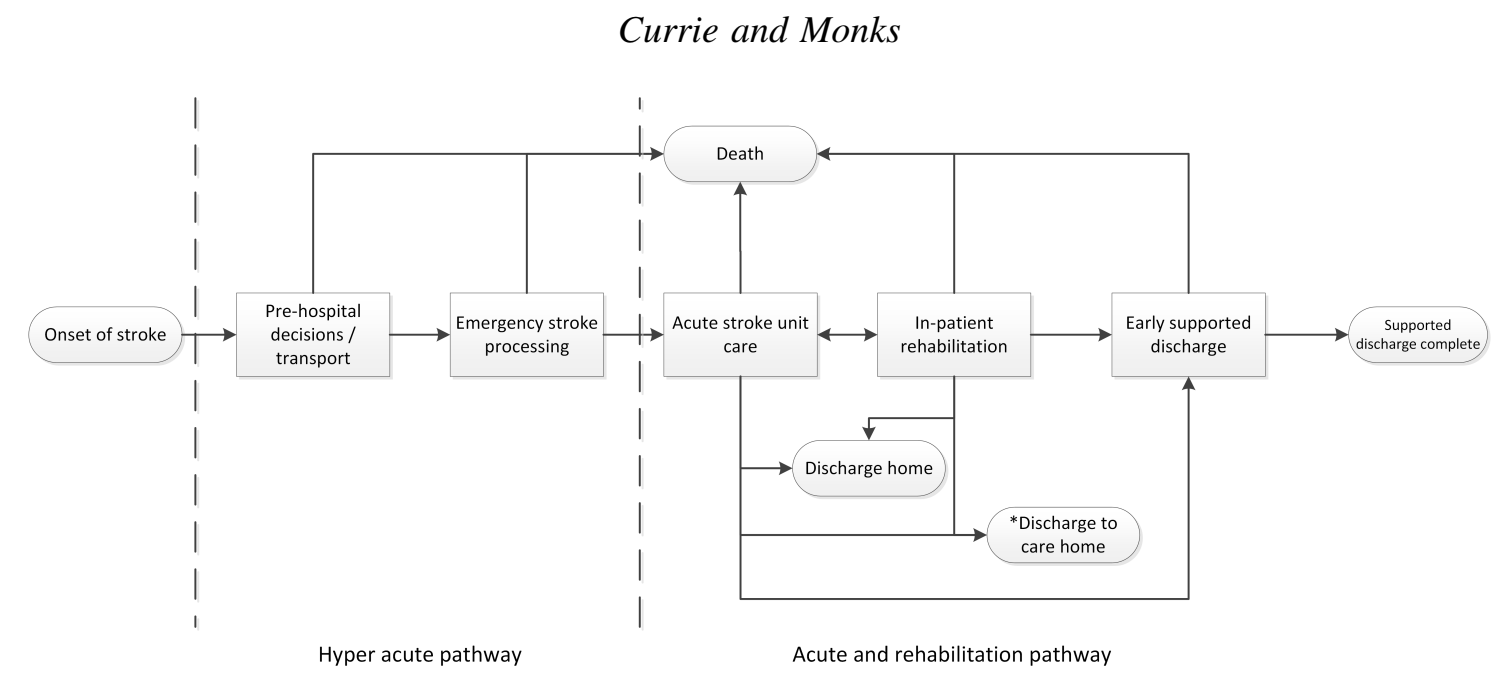

Figure 3: Overview of acute stroke and rehabilitation pathway.

minority of cases a patients condition may deteriorate so they bounce-back from rehabilitation to acute care units. The difficulty in discharge from rehabilitation wards can lead to so called bed-blocking or in more familiar terms queueing; meaning operational difficulty for any upstream ASU.

Both acute and rehabilitation wards make use of early supported discharge (ESD) services which can be made up of both therapists and nursing staff.

\subsection{Study Setting}

In early 2014, a hospital in the UK approached a modeling team to assist with planning the capacity within a hospital-based service that treated patients with acute symptoms of stroke and managed their long-term rehabilitation. The hospital was performance managed on the proportion of stroke patients that were admitted to the Acute Stroke Unit (ASU) within four hours. The hospital had been struggling to meet this target for some time. To manage lengthy admission delays the hospital had to make additional transfers of patients between wards. All these moves required a porter, a nurse and completion of associated admission and transfer paperwork.

\subsection{Input Data}

The model was constructed using administrative data collected by the acute and community services. The patient population included diagnoses of stroke, complex neurological conditions and general medical patients who were forced to use the ASU when their specialist wards were full.

\subsection{Simulation Model}

The modeling team were given a short deadline to support a business case for changing the configuration of the system to reduce admission delays. A DES model was developed. Full details of this study can be found in (Monks et al. 2016). Here we focus on the simplest aspect: the number of beds required in different settings. The study team faced a choice of modeling the queuing within the stroke pathway in detail, i.e. including the escalation logistics described above, or to provide a simpler model that was gave indicative estimates of appropriate bed capacity to reduce waiting times. Given the time constraints of the project, the team opted to use the unfettered demand approach introduced by (Utley and Worthington 2012). Instead of attempting to model this management in detail, capacity constraints can be removed from a model (an infinite server approach). Infinite server simulation models predict the probability that a patient will be rejected (blocked) from a ward and eliminates the need to understand management of patients when capacity is insufficient. Such models are different from the real system, but are still a valuable decision 
aid for capacity planning. Verification and validation made use of standard procedures (Sargent 2013); for example, structured walk through of the conceptual and coded model with clients, and independent code reviews.

\subsection{Use of the Model}

Figure 4 illustrates how the model was used with the clinicians and management of the stroke pathway. The model was first used to illustrate simulated occupancy (Figure 4) and highlight the most likely range (middle 90\%) of beds that could be in use. The second stage converted the simulated occupancy to the probability that a patient is delayed. When combined both results allowed the clients to see why the current bed stock (10) led to a large number of patient transfers and the diminishing returns increasing beds beyond 13 or 14. Uncertainty in model outputs was explored using sensitivity analysis of input distribution parameters.

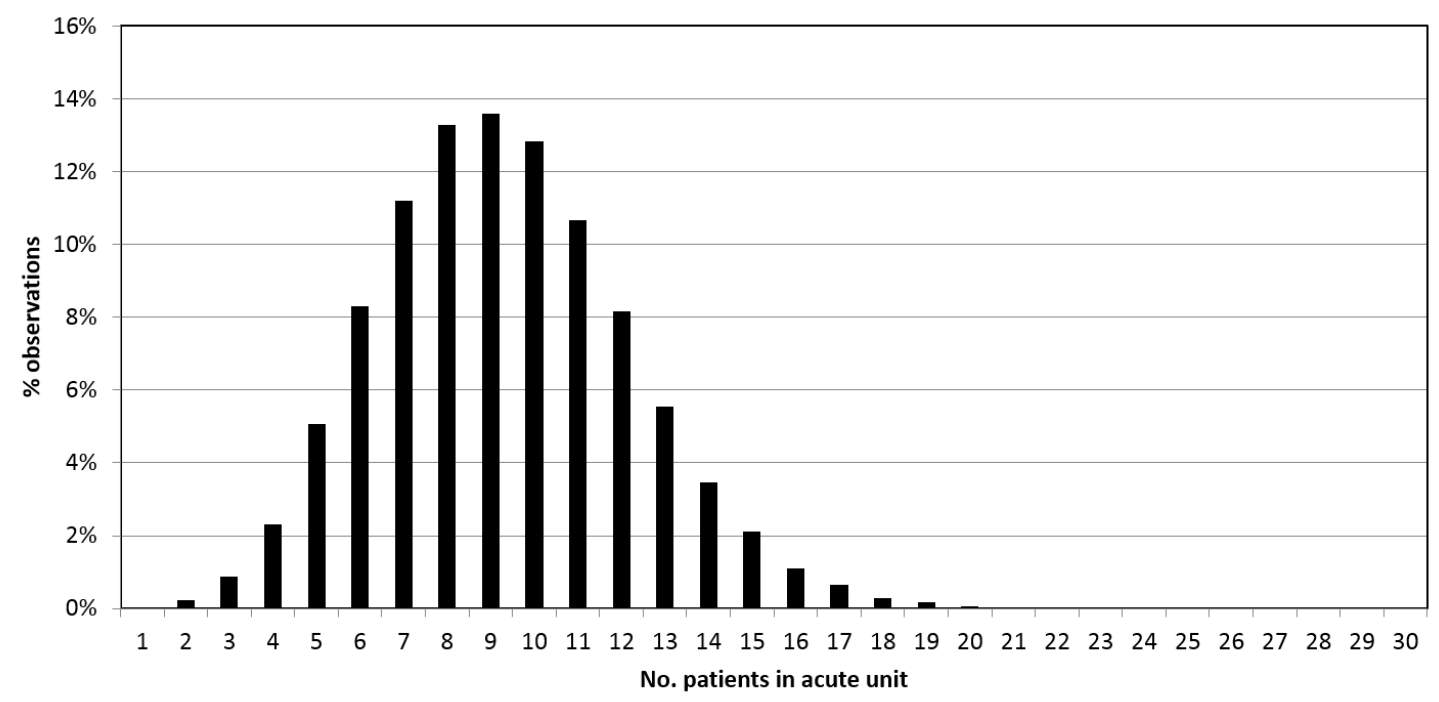

Figure 4: Stroke Ward Simulated Occupancy.

\section{CONCLUSION}

It seems inevitable that diseases will continue to be of importance to the human population for the foreseeable future and as modeling methods improve and the volume of data available increases, the models we build of diseases will become more sophisticated. This does present some methodological challenges, particularly with regard to calibrating multiple model parameters based on limited (and possibly inaccurate) observational data.

While there is a considerable body of work in the area, we can identify a few open questions that we anticipate future research considering.

Much of the work considered in previous models, particularly with regard to non-communicable diseases, is concerned with coping with a given level of demand. Perhaps a key future question is how best to reduce that level of demand, e.g., how best to target interventions that improve lifestyle.

Many of the reductions in transmission of infectious diseases has come about as a result of poverty reduction. For example, diseases such as TB which rely on close contact for transmission can be reduced significantly by reducing overcrowding. Improved sanitation has also helped to reduce the levels of many health conditions (Lancet 2014). Being able to assess the full benefits of such non-specific interventions could also help international agencies to target their resources most effectively. 


\section{Currie and Monks}

Prevention of non-communicable disease involves identifying patients most at risk of developing the disease at the same time as constraining costs. For example, in the stroke domain as many as $15 \%$ of all strokes are caused by patients with an irregular heartbeat called atrial fibrillation (AF). Many people with AF are unaware of their condition and their eventual diagnosis occurring after a stoke has occurred (Dewar and Lip 2007). Modeling could therefore investigate competing screening strategies for AF and its impact on wider stroke pathways operations and longer-term costs. This might include opportunistic screening strategies, for example, when people are screened for known co-morbidities such as hypertension or diabetes.

The article demonstrates a rise in the use of ABMs for analysis of communicable diseases and the increased size and complexity of the disease models currently being built. Recent large-scale modeling studies draw on specialists from a range of different areas such as ethnography and the social sciences as well as medical experts and mathematicians and computer scientists. Such big teams of people with diverse interests can generate new insights but care needs to be taken not to lose sight of the benefits of simple models. Including a great deal of detail into a model requires both large quantities of data and time-consuming sensitivity analyses of the model parameters in order to justify the models conclusions. Conversely, the more detailed a model the more realistic it can seem, which can make it more acceptable to users.

\section{REFERENCES}

Aleman, D. M., T. G. Wibisono, and B. Schwartz. 2009. "Accounting for individual behaviors in a pandemic disease spread model". In Proceedings of the Winter Simulation Conference 2009, 1977-1985.

Anderson, R. M., and R. M. May. 1993. Infectious diseases of humans: dynamics and control. Oxford, UK: Oxford University Press.

Bailey, N. 1950. "A simple stochastic epidemic". Biometrika 37:193-202.

Bartlett, M. 1957. "Some evolutionary stochastic processes". Journal of the Royal Statistical Society, Series B 111:211-229.

Benneyan, J., J. Garrahan, I. Ilieş, and X. Duan. 2017. "Modeling approaches, challenges and preliminary results for the opioid and heroin co-epidemic crisis". In Proceedings of the Winter Simulation Conference 2017, 2821-2832.

Brailsford, S., P. Harper, and J. Sykes. 2012. "Incorporating human behaviour in simulation models of screening for breast cancer". European Journal of Operational Research 219(3):491 - 507.

Cepoiu-Martin, M., and D. P. Bischak. 2017. "Policy choices in dementia care. An exploratory analysis of the Alberta continuing care system (ACCS) using system dynamics". Journal of Evaluation in Clinical Practice 24(1):278-284.

Chalk, D., M. Pitt, B. Vaidya, and K. Stein. 2012. "Can the Retinal Screening Interval Be Safely Increased to 2 Years for Type 2 Diabetic Patients Without Retinopathy?". Diabetes Care 35(8):1663-1668.

Codeço, C. T., M. P. Subhash Lele, M. Bouma, and A. I. Ko. 2008. "A stochastic model for ecological systems with strong nonlinear response to environmental drivers: application to two water-borne diseases". Journal of the Royal Society Interface 5:247-252.

Comas, M., J. Mendivil, M. Andreu, C. Hernndez, and X. Castells. 2016, 10. "Long-Term Prediction of the Demand of Colonoscopies Generated by a Population-Based Colorectal Cancer Screening Program". PLOS ONE 11(10):1-13.

Crooks, A., and A. Hailegiorgis. 2013. "Disease modeling within refugee camps: a multi-agent systems approach". In Proceedings of the Winter Simulation Conference 2013, 1697-1706.

Davies, R., S. Brailsford, P. Roderick, C. Canning, and D. Crabbe. 2000, Apr. "Using simulation modelling for evaluating screening services for diabetic retinopathy". Journal of the Operational Research Society 51(4):476-484. 


\section{Currie and Monks}

de Cock, K. M., and R. E. Chaisson. 1999. "Will DOTS do it? A reappraisal of tuberculosis control in countries with high rates of HIV infection". The International Journal of Tuberculosis and Lung Disease 3:457-465.

de Lima, T. F. M., R. M. Lana, T. G. de Senna Carneiro, C. T. Codeço, G. S. Machado, L. S. Ferreira, L. C. de Castro Medeiros, and C. A. D. Junior. 2016. "DengueME: A Tool for the Modeling and Simulation of Dengue Spatiotemporal Dynamics". International Journal of Environmental Research and Public Health 13:920.

der Ploeg, C. P. B. V., C. V. Vliet, S. J. D. Vlas, J. O. Ndinya-Achola, L. Fransen, G. J. V. Oortmarssen, and J. D. F. Habbema. 1998. "STDSIM: a microsimulation model for decision support in STD control". Interfaces 28:84-100.

Dewar, R. I., and G. Y. H. Lip. 2007. "Identification, diagnosis and assessment of atrial fibrillation". Heart 93(1):25-28.

Enns, E. A., and M. L. Brandeau. 2011. "Inferring model parameters in network-based disease simulation". Health Care Management Science 14:174-188.

Epstein, J. M. 2009. "Modelling to contain pandemics". Nature 460:687.

Harper, P. R., and A. K. Shahani. 2003. "A decision support system for the care of HIV and AIDS patients in India". European Journal of Operational Research 147:187-197.

Hu, S. X., S. Barnes, and B. Golden. 2014. "Early detection of bioterrorism: monitoring disease using an agent-based model". In Proceedings of the Winter Simulation Conference 2014, 310-321.

Isidoro, C., N. Fachada, F. Barata, and A. Rosa. 2011. "Agent-Based Model of Dengue Disease Transmission by Aedes aegypti Populations". In Advances in Artificial Life. Darwin Meets von Neumann. ECAL 2009. Lecture Notes in Computer Science, vol 5777, 345-352.

Jansen, J., and J. Zoetelief. 1997. "Optimisation of mammographic breast cancer screening using a computer simulation model”. European Journal of Radiology 24(2):137 - 144.

Jung, H.-J., G.-S. Jung, Y. Kim, N. T. Khan, Y.-H. Kim, Y.-B. Kim, and J.-S. Park. 2017. "Development and application of agent-based disease spread simulation model: the case of Suwon, Korea". In Proceedings of the Winter Simulation Conference 2017, 2810-2820.

Kasaie, P., J. R. Andrews, W. D. Kelton, and D. W. Dowdy. 2014. "Timing of Tuberculosis Transmission and the Impact of Household Contact Tracing". American Journal of Respiratory Critical Care Medicine 189:845-852.

Kasaie, P., D. W. Dowdy, and W. D. Kelton. 2013. "An agent-based simulation of a tuberculosis epidemic: understanding the timing of transmission". In Proceedings of the Winter Simulation Conference 2013, 2227-2238.

Keeling, M., and P. Rohani. 2007. Modeling Infectious Diseases in Humans and Animals. Princeton, USA: Princeton University Press.

Kenyon, T. A., S. E. Valway, W. W. Ihle, I. M. Onorato, and K. G. Castro. 1996. "Transmission of multidrug-resistant mycobacterium tuberculosis during a long airplane flight". New England Journal of Medicine 334:933-938.

Kermack, W. O., and A. G. McKendrick. 1933. "Contributions to the mathematical theory of epidemics. III. Further studies of the problem of endemicity". Proceedings of the Royal Society of London, Series A 141:94-112.

Knerer, G., C. S. M. Currie, and S. C. Brailsford. 2015. "Impact of combined vector-control and vaccination strategies on transmission dynamics of dengue fever: a model-based analysis". Health Care Management Science 18:205-217.

Kok, S., A. R. Rutherford, R. Gustafson, R. Barrios, J. S. G. Montaner, and K. Vasarhelyi. 2015. "Optimizing an HIV testing program using a system dynamics model of the continuum of care". Health Care Management Science 18:334-362. 


\section{Currie and Monks}

Lahr, M. M. H., D.-J. van der Zee, G.-J. Luijckx, P. C. A. J. Vroomen, and E. Buskens. 2017, Jan. "Centralising and optimising decentralised stroke care systems: a simulation study on short-term costs and effects". BMC Medical Research Methodology 17(1):5.

Lancet 2014. "Water and sanitation: addressing inequalities (editorial)". The Lancet 383:1359.

Lebcir, R., E. Demir, R. Ahmad, C. Vasilakis, and D. Southern. 2017, Jan. "A discrete event simulation model to evaluate the use of community services in the treatment of patients with Parkinson's disease in the United Kingdom". BMC Health Services Research 17(1):50.

Lee, T., H.-R. Lee, and K. Hwang. 2013. "Identifying superspreaders for epidemics using R0-adjusted network centrality". In Proceedings of the Winter Simulation Conference 2013, 2239-2249.

Long, E. F., N. K. Vaidya, and M. L. Brandeau. 2008. "Controlling co-epidemics: analysis of HIV and tuberculosis infection dynamics". Operations Research 56:1366-1381.

Ma, Y., K. Bisset, J. Chen, S. Deodhar, and M. Marathe. 2011. "Efficient implementation of complex interventions in large scale epidemic simulations". In Proceedings of the Winter Simulation Conference 2011, 1354-1366.

Mandal, S., R. R. Sarkar, and S. Sinha. 2011. "Mathematical models of malaria - a review". Malaria Journal 10:202.

Mellor, G. R., C. S. Currie, and E. L. Corbett. 2010. "Incorporating Household Structure into a DiscreteEvent Simulation Model of Tuberculosis and HIV". ACM Transactions on Modeling and Computer Simulation 21:26.

Monks, T., D.-J. van??der??Zee, M. M. Lahr, M. Allen, K. Pearn, M. A. James, E. Buskens, and G.-J. Luijckx. 2017. "A framework to accelerate simulation studies of hyperacute stroke systems". Operations Research for Health Care 15:57 - 67.

Monks, T., D. Worthington, M. Allen, M. Pitt, K. Stein, and M. A. James. 2016, Sep. "A modelling tool for capacity planning in acute and community stroke services". BMC Health Services Research 16(1):530.

Murray, M. 2002. "Determinants of cluster distribution in the molecular epidemiology of tuberculosis". Proceedings of the National Academy of Sciences 99:1538-1543.

Mustafee, N., T. Lyons, P. Rees, L. Davies, M. Ramsey, and M. Williams. 2012, 12. "Planning of bed capacities in specialized and integrated care units: Incorporating bed blockers in a simulation of surgical throughput". 1-12.

Okhmatovskaia, A., A. Verma, B. Barbeau, A. Carriere, R. Pasquet, and D. Buckeridge. 2010. "A Simulation Model of Waterborne Gastro-Intestinal Disease Outbreaks: Description and Initial Evaluation". In AMIA Annual Symposium Proceedings, 557-561.

Özaltin, O. Y., Özden O. Dalgic, and F. S. Erenay. 2014. "Optimal distribution of the influenza vaccine". In Proceedings of the Winter Simulation Conference 2014, 1411-1420.

Paleshi, A., G. W. Evans, S. S. Heragu, and K. S. Moghaddam. 2011. "Simulation of mitigation strategies for a pandemic influenza". In Proceedings of the Winter Simulation Conference 2011, 1345-1353.

Paneth, N., P. Vinten-Johansen, H. Brody, and M. Rip. 1998. "A rivalry of foulness: official and unofficial investigations of the London cholera epidemic of 1854". American Journal of Public Health 88:15451553.

Perkins, A., R. Reiner, G. España, Q. ten Bosch, A. Verma, K. Liebman, V. Paz Soldan, J. Elder, A. C. Morrison, S. Stoddard, U. Kitron, G. M. Vazquez-Prokopec, T. W. Scott, and D. L. Smith. 2018. "An agent-based model of dengue virus transmission shows how multiple uncertainties about vaccine efficacy influence public health impact projections". bioRxiv.

Ross, R. 1911. The prevention of malaria. London, UK: John Murray.

Rutherford, A. R., B. Ramadanović, L. Ahrenberg, W. Michelow, B. D. L. Marshall, W. Small, K. Deering, J. S. G. Montaner, and K. Vasarhelyi. 2016. "Control of an HIV epidemic among injection drug users: simulation modeling on complex networks". In Proceedings of the Winter Simulation Conference 2016, $23-37$. 
Sanchez, P. J., and S. M. Sanchez. 2015. "A scalable discrete event stochastic agent-based model of infectious disease propagation". In Proceedings of the Winter Simulation Conference 2015, 151-158.

Sargent, R. G. 2013. "Verification and validation of simulation models". Journal of Simulation 7(1):12-24. Song, E., B. L. Nelson, and C. D. Pegden. 2014, Dec. "Advanced tutorial: Input uncertainty quantification". In Proceedings of the Winter Simulation Conference 2014, 162-176.

Thompson, J. P., C. M. Riley, and R. L. Eberlein. 2014. "Modelling for Insight: The Case of Dementia in Singapore". Systems Research and Behavioral Science 31(2):227-235.

Utley, M., and D. Worthington. 2012. "Capacity Planning". In Handbook of Healthcare System Scheduling, edited by R. Hall. New York: Springer.

Vasilakis, C., B. G. Sobolev, L. Kuramoto, and A. R. Levy. 2007, Feb. "A simulation study of scheduling clinic appointments in surgical care: individual surgeon versus pooled lists". Journal of the Operational Research Society 58(2):202-211.

Worth, T., R. Uzsoy, E. Samoff, A.-M. Meyer, and J.-M. Maillard. 2010. "Modeling the response of a public health department to infectious disease". In Proceedings of the Winter Simulation Conference 2010, 2185-2198.

Zhang, T., X. Fu, M. Lees, C. K. Kwoh, G. K. K. Lee, and R. S. M. Goh. 2012. "A contact-network-based simulation model for evaluating interventions under "what-if" scenarios in epidemic". In Proceedings of the Winter Simulation Conference 2012, 3833-3844.

CHRISTINE CURRIE is Associate Professor of Operational Research in Mathematical Sciences at the University of Southampton, UK, where she also obtained her Ph.D. She is Editor-in-Chief for the Journal of Simulation. Christine co-chaired the 9th UK Simulation Workshop, SW18. Her research interests include mathematical modeling of epidemics, Bayesian statistics, revenue management, variance reduction methods and optimization of simulation models. Her website address is http://www.southampton.ac.uk/maths/about/ staff/ccurrie.page and her email address is Christine.Currie@soton.ac.uk.

THOMAS MONKS is the Director of Data Science at NIHR CLAHRC Wessex, University of Southampton. His research interest is applied simulation modelling, optimization and machine learning in healthcare. His email address is thomas.monks@ soton.ac.uk and his ORCID is orcid.org/0000-0003-2631-4481 